FEDSM2017-69272

\title{
MACHINE LEARNING CONTROL FOR EXPERIMENTAL TURBULENT FLOW TARGETING THE REDUCTION OF A RECIRCULATION BUBBLE
}

\author{
Camila CHOVET * \\ Marc LIPPERT \\ Laurent KEIRSBULCK \\ LAMIH UMR CNRS 8201 \\ University of Valenciennes \\ and Hainaut-Cambresis \\ Campus du Mont Houy \\ F-59300, France
}

\author{
Bernd R. NOACK ${ }^{\mathrm{a}, \mathrm{b}}$ \\ a LIMSI-CNRS UPR 3251 \\ Campus University of Orsay \\ F-91405 Orsay, France \\ b Institut für Strömungsmechanik \\ Technische Universität Braunschweig \\ D-38108 Braunschweig, Germany
}

\author{
Jean-Marc FOUCAUT \\ LML UMR CNRS 8107 \\ Ecole Centrale de Lille \\ France
}

Email: camila.chovet@etu.univ-valenciennes.fr

\begin{abstract}
We experimentally control the turbulent flow over backwardfacing step $\left(\operatorname{Re}_{H}=31500\right)$. The goal is to modify the internal $(\mathrm{Xr})$ and external $(\mathrm{Lr})$ recirculation points and consequently the recirculation zone (Ar). A model-free machine learning control (MLC) is used as control logic. As benchmark, an optimized periodic forcing is employed. MLC generalizes periodic forcing by a multi-frequency actuation. In addition, a sensor-based control and a non-autonomous feedback, open- and closed-loop laws, were use to optimize the control. The MLC multi-frequency forcing outperforms, as expected, periodic forcing. The nonautonomous feedback brings a further improvement. The unforced and actuated flows have been investigated in real-time with a TSI particle image velocimetry (PIV) system. The current study shows that a generalization of multi-frequency forcing and sensor feedback significantly reduces the turbulent recirculation zone, far beyond optimized periodic forcing. The study suggests that MLC can effectively explore and optimize new feedback actuation mechanisms and we anticipate MLC to be a game changer in turbulence control.
\end{abstract}

* Address all correspondence to this author.

\section{KEYWORDS}

Machine learning control, experimental flow control, recirculation zone.

\section{NOMENCLATURE}

Ar Recirculation area.

b Control law candidate.

$\delta$ Boundary layer.

$\mathrm{f}$ Frequency.

H Step height.

i MLC individual.

J Cost function.

Lr External separation point.

$\lambda$ Actuation cost.

$\mathrm{n}$ MLC generation.

P Pressure probe.

Re Reynolds number.

$U_{0}$ Free stream velocity.

$u_{\text {jet }}$ Free stream velocity.

$T_{e} \quad$ MLC evaluation time.

$\mathrm{s}$ Sensor.

$S t_{H} \quad$ Strouhal number.

$\mathrm{Xr}$ Internal separation point. 


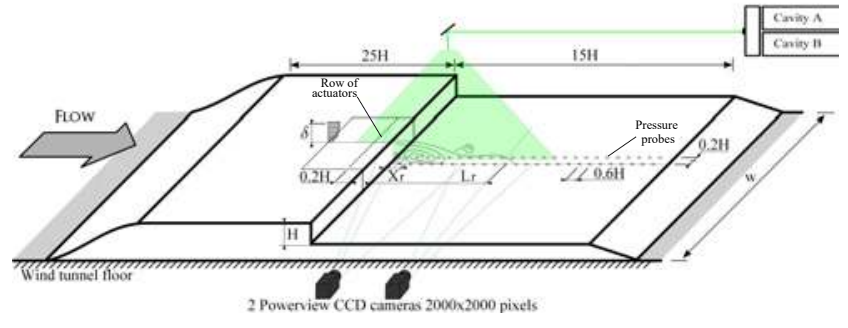

FIGURE 1. SCHEMATIC OF THE EXPERIMENTAL SETUP

\section{INTRODUCTION}

Flow with separation and reattachment has long been a subject of fundamental fluid dynamics research. This flow gives rise to unsteadiness, pressure fluctuations, structure vibrations and noise [1]. The flow over a backward-facing step (BFS) is a common separating/reattaching flow found in nature. Therefore is deeply studied both numerically and experimentally (see [1-4]). The control of separated and reattaching flows is an essential issue for practical applications. Periodically perturbing the shear layer at the separation location using synthetic jet actuator was found to be an effective tool to control these flows [5-8]. Bhattacharjee et al. [9] claimed that the most effective non-dimensional forcing frequency, so called Strouhal Number $\left(S t_{H}=f * H / U_{0}\right)$, was between 0.2 and 0.4. Closed-loop control offers further possibilities to improve the actuation efficiency by adapting the control to different flow conditions. There are two main ways to generate the closed-loop control laws. The first one is to compute the model using previous knowledge of the physics of the flow [10]. But, these models, obtained from the derivation of the NavierStokes equations, are of very high order and require reduction before they can be used in a realistic settings [11]. The challenges of model-based control design have led us to inclined to model-free control strategies using machine learning methods such as genetic programming (GP). GP is a powerful regression method which can detect and exploit nonlinear actuation mechanisms in an unsupervised manner, as evidenced in several shear flow control experiments [11-13].

The goal of this study is to use the performance benefits of machine learning control MLC in active flow control to find a cost effective control law. The control objective is to reduce the recirculation zone downstream of a backward-facing step (BFS). Particle image velocimetry PIV will be employed to the physical mechanisms of turbulent unperturbed and perturbed flows.

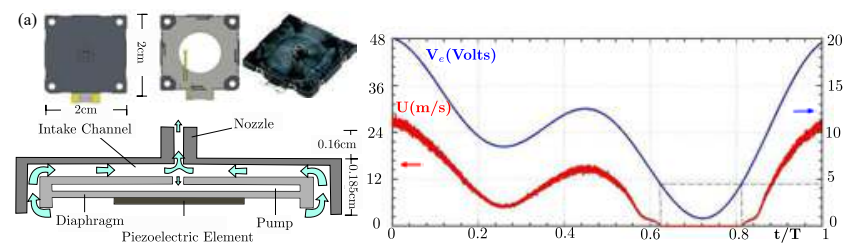

FIGURE 2. ACTUATION SYSTEM. a) PULSED JET SCKETCH; b) VELOCITY RESPONSE TO AN ARBITRARY SIGNAL

\section{EXPERIMENTAL APPARATUS AND TECHNIQUES Description of the test facility and the step}

The experiments were conducted in an optically accessible closed-loop wind tunnel. The test section is $2 \mathrm{~m}$ wide, $2 \mathrm{~m}$ high and $10 \mathrm{~m}$ long. The free stream velocity is $U_{0}=5.7 \mathrm{~m} / \mathrm{s}$, $R e_{H}=31500\left(\delta=0.68 H, R e_{\delta}=21500\right)$. The air is conditioned in a settling chamber to minimize free stream turbulence through a contoured converging nozzle. The model is mounted in the middle of the test section. The step height of the backwardfacing step is $H=83 \mathrm{~mm}$ and corresponds to an expansion ratio of 1.04, i.e. negligible inference of the upper wall. An effectively nominally two-dimensional flow is provided by the large span of $2000 \mathrm{~mm}$ yielding an aspect ratio of 24 [14]. The array of actuators was placed at the middle the test section width and is $5 H$ long (20\%), leaving a free space of $800 \mathrm{~mm}$ between the control area and the test section walls. This prevents flow interactions between the corner of the test section and the backward facing step. The origin of the coordinate system is located at the edge of the step. The flow is described in a Cartesian coordinate system with $\mathrm{x}, \mathrm{y}, \mathrm{z}$ representing streamwise, spanwise (or lateral) and transverse (or normal to ground) directions, respectively. The setup described above is illustrated in a $3 \mathrm{D}$ view in FIG. 1.

\section{Actuation system}

The actuation system is driven by 20 piezoelectric devices (Murata micro-blowers, see FIG. 2(a)) which distribute the air flow through micro-nozzles (micro-pulsed jets). The distance between two neighboring micro-nozzles is $20 \mathrm{~mm}$. The location of the actuators was chosen in agreement with literature and previous works [11] The micro-jets axes are perpendicular to the wall as seen in FIG. 1.

Contrary to other classical pulsed jets devices, Murata micro-blowers only need a power supply of 5 to 20 Volts. The mean jet velocity $\left(u_{\text {jet }}\right)$ provided by each micro-blower is expected to range between $0 \mathrm{~m} / \mathrm{s}$ to $30 \mathrm{~m} / \mathrm{s}$ and is proportional to the voltage command up to $100 \mathrm{~Hz}$. The jet velocity can be commanded over a wide range of frequencies with large accuracy, avoiding, for instance, dynamic overshoots citeCHO16. As an example FIG. 2(b) shows the actuator re- 
sponse to a complex input voltage signal. These actuators have unique advantages for closed-loop flow control applications.

\section{Pressure Measurements}

Recirculation length reduction is highly correlated with the pressure from which the control performance can be quantified. A set of 25 static pressure taps were distributed downstream of the back-facing step in the freestream direction. The pressure tabs were mounted starting at $25 \mathrm{~mm}$ with an interval between each other of $50 \mathrm{~mm}\left(x_{i} / H=0.6\right)$, as shown in FIG. 1. In addition, a second set of 25 sub-miniature piezo-resistive Kulite XCQ-062 sensors with a nominal measurement range of $35 \mathrm{kPa}$. Flush-mounted Kulite transducers were placed in parallel and at the same $x_{i}$ location as the static ones. Thus, the 25 unsteady and static pressure sensor arrays extents up to $14.75 \mathrm{H}$ in the streamwise direction.

\section{Velocity measurements}

Velocity measurements were performed downstream of the step by the use of standard two-component TSI particle image velocimetry (PIV). The PIV field is of view is about $7.3 \mathrm{H} \times 1.8 \mathrm{H}$ on the $x-y$ plane passing through center of the backward facing step. This large field spans the whole wake containing entirely the recirculating flow domain. The flow is seeded with oil particles using a jet atomizer upstream the stagnation chamber. The atomizer location allows homogenous dispersion of the particles throughout the test section.

The system consists of a double-pulse laser system and two cameras $(2000 \times 2000$ pixels charge-coupled-device Powerview with a $50 \mathrm{~mm}$ optical lens). The frequency-doubled laser (Qswitched Nd:YAG operating at $532 \mathrm{~nm}$; dual-head BigSky) emits laser pulses with a maximum energy of $200 \mathrm{~mJ}$. The resolution is 19.2 pixels $/ \mathrm{mm}$ for the employed cameras. The dynamic range is approximately 30 pixels and the velocity vectors are processed with an interrogation window $16 \times 16$ pixels with an overlap of $50 \%$. For every flow/actuation configurations, 2000 doubleframe pictures are recorded to ensure the velocity fields statistics convergence. The PIV time-uncorrelated snapshots are recorded with a repetition rate of $7 \mathrm{~Hz}$. The velocity measurements are sampled at $10 \mathrm{kHz}$ and latter filtered at $3 \mathrm{kHz}$.

\section{GENETIC PROGRAMMING CONTROL}

Duriez et al. [16] formulated a generic, model-free, approach to open- and closed-loop control of nonlinear systems called Machine learning control (MLC) . The control design is formulated as a regression problem: find the control law which optimizes a given cost function. An initial population of control law candidates $\left(b_{i}^{(1)}\left(s_{j}\right)\right)$, called individuals, is generated randomly by combining user-defined functions, constants and sensors $s_{j}$ (or signals); like in a Monte-Carlo method. Each individ- ual is graded in the experiment (inner loop) and a cost function $J_{1}^{(1)}$ is attributed to them. Each generation is made of 100 individuals to assure variability. A new population of individuals $b_{i}^{(2)}$ is then evolved from the previously evaluated one by genetic operators, leading to new cost functions $J_{i}^{(2)}$. Without loss of generality, the control laws of the $n$-generation are ordered $J_{1}^{(n)} \leq J_{2}^{(n)} \leq \ldots \leq J_{100}^{(n)}$. This learning process will continue until some stopping criterion is met. In the present study a the number of generations was set to 12 , which was the minimum amount of generations required to converge toward the best individual. The targeted optimal control law is the best individual of the last generation. This individual is taken as MLC law. This marks the end of the learning phase. This process is summarized in FIG. 3.

Three different approaches for control laws are considered. First, multi-frequency control $b=k\left(h_{j}\right)$ generalizes periodic forcing using three sine signals at $14 \mathrm{~Hz}, 15 \mathrm{~Hz}$ and $16 \mathrm{~Hz} ; \mathrm{St}_{H}$ of $0.211,0.226$ and 0.241 , as inputs. These frequencies correspond to the best periodic forcing frequencies obtained in previous experiences. Second, in sensor-based control $b=k\left(P_{j}\right)$, the pressure probes are used to generate the control laws. Finally, multi-frequency forcing and sensor-based feedback is generalized in the non-autonomous law $b=k\left(P_{j}, h_{j}\right)$, i.e., a combination of harmonic and sensor-based inputs is taken.

As the main goal is to reduce the external separation point $\left(L_{r}\right)$, The cost function $J=L_{r}^{E}+\lambda<b>$ was based on an estimated $L_{r}^{E}$ from a quadratic fit of the spatial pressure fluctuation distribution $C p_{r m s}$. This was achieved using four pressure probes $\left(P_{7}, P_{8}\right.$, $\left.P_{10} \& P_{13}\right)$ with an evaluation time of $T_{e}=15 \mathrm{~s}$. The penalization parameter prioritize either the separation length reduction $(\lambda<1)$ or the actuation $\cos t(\lambda>1)$. A penalization $\lambda=0.1$ was set based on the low energy consumption of the row of micro-blower.

\section{RESULTS AND DISCUSSION}

To obtain the external and internal reattachment lengths and consequently the recirculation area, Simpsons et al. estimates the points where the backward flow probability (BFP) reaches a value of $50 \%$ [17]. To actively control the flow of the backwardfacing step, experiments were carried out varying the frequency $(f)$ of the periodic actuation commands $\left(b=U_{j e t} \operatorname{Sin}(2 p i f t)\right)$, where $U_{\text {jet }}$ is the velocity actuation amplitude set constant at $25 \mathrm{~m} / \mathrm{s}$ (see FIG. 5(h)). Both internal (Xr) and external (Lr) separation points and the separation area (Ar) as a function of the Strouhal Number $S t_{H}=f * H / U_{0}$ are shown in Fig. 4. The reattachment lengths and area are normalized by those of the unperturbed experiment. Xr increases while $\mathrm{Lr}$ and $\mathrm{Ar}$ decreased with increasing $S t_{H}$ reaching positive and negative peaks at $S t_{H}=0.226$, respectively. From results, the best forcing frequency was observed at $f=15 \mathrm{~Hz}, S t_{H}=0.226$ and is associated with the shedding phenomena. Xr increases up to $38.4 \%$ while Lr and Ar decrease to $4.8 \%$ and $19.0 \%$, respectively. The results 


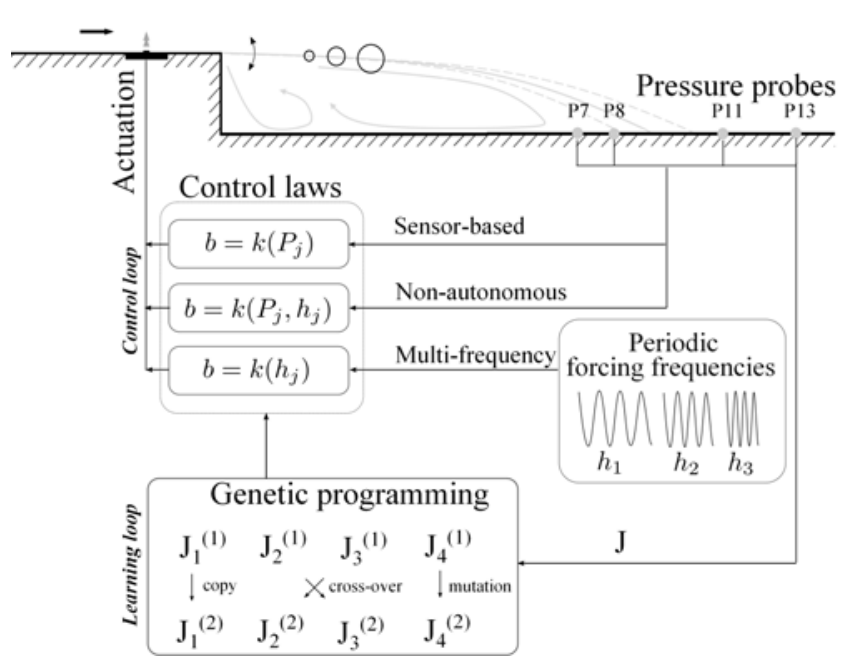

FIGURE 3. GENETIC PORGRAMMING CONTROL LOOP SKETCH

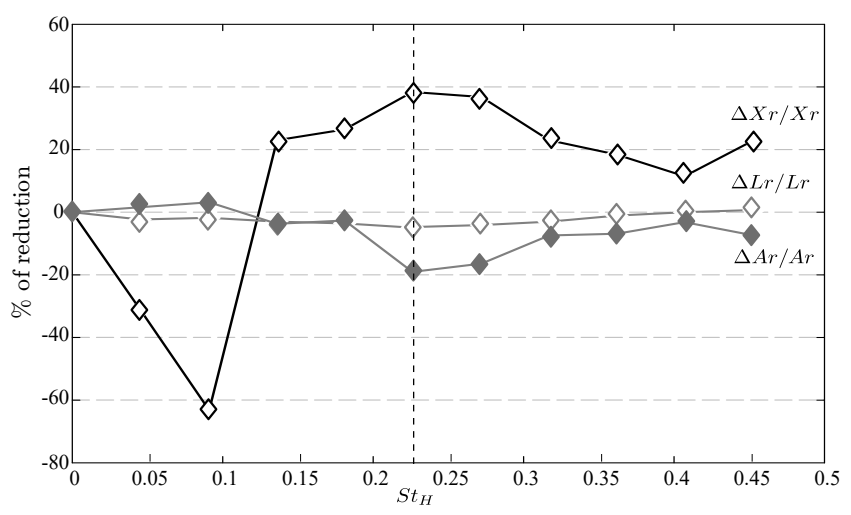

FIGURE 4. RECIRCULATION PARAMETERS AS A FUNCTION OF THE STROUHAL NUMBER. $\diamond$ INTERNAL SEPARATION POINT Xr, $\diamond$ EXTERNAL SEPARATION POINT Lr, $\diamond$ RECIRCULATION AREA Ar. VARIATIONS ARE IN \%

of the present study are consistent with those obtained in previous experiments $[5,6,9,18]$. PIV measurements were performed at the optimal frequency, the BFP obtained is shown in FIG. 5(d). This corroborates the effectiveness of pulsing jet injection with forcing frequency to reduce the separation length [19].

MLC studies were carried out to discover new and more effective closed-loop separation control laws. Three cases were studied: multi-frequency open-loop, sensor-based and nonautonomous control laws. After 12 generations with 100 individuals, PIV measurements were carried out for the optimal control; represented in FIG. 5(g), (i) and (j) for sensor-based, multifrequency open-loop and non-autonomous, respectively. The
BFP values extracted at the vicinity of the wall $(y / H=0.07)$ are plotted in FIG. 5(a) for: unforced (dot-dashed gray line), periodic forcing (solid gray line), multi-frequency open-loop (dashed black line), sensor-based (dashed gray line) and non-autonomous control (solid black line). The increase of the internal separation length and the decrease of the external separation point are clearly seen from the unforced flow (dot-dashed gray line) to the non-autonomous control (solid black line). It is interesting to note that multi-frequency open-loop (dashed black line) out-performs the periodic forcing, but the sensor-based (dashed gray line) control law is the least efficient. Moreover, to better understand the effects of the different control laws over the flow, the BFP fields for all cases are presented in FIG. 5(bf). Periodic forcing BFP field is seen in FIG. 5(d). For the multi-frequency open-loop case (Fig. 5(e)), a higher reduction of the external separation point $\left(L_{r}=5.18 H\right)$, compared to the best forcing frequency, is observed and an increase in the internal separation point $X_{r}=1.42 \mathrm{H}$ can be seen. On the contrary, for the sensor-based case (FIG. 5(c)), the internal and external separation point did not change significantly. We obtained $L_{r}=5.55 \mathrm{H}$ and $X_{r}=0.95 \mathrm{H}$. As expected, the best case combines sensor-based feedback and open-loop forcing. The resulting non-autonomous feedback (FIG. 5(f)) gave a strong reduction of the external separation point up to $8.6 \%\left(L_{r}=5.13 H\right)$ and an increase of $70.6 \%$ of the internal separation point $X_{r}=1.48 H$. Same results are seen for the recirculation area, where the best control law is the MLC non-autonomous reducing this region up to $40.7 \%$. The current study shows that a generalization of multifrequency forcing and sensor feedback control law significantly reduces the turbulent recirculation zone. A summary of all the results is presented in FIG. 6. The key enabler is MLC for optimizing the control laws. The study indicates that MLC can effectively explore and optimize new feedback actuation mechanisms in numerous experimental applications.

\section{CONCLUSION}

Experimental flow control in a separated shear layer using micro-pulsed jets was done. The control target was to modify key turbulent flow parameters such as: internal separation point, external separation point and recirculation area, more specifically increase the first parameter and reduce the others. Periodic forcing, open- and closed-loop machine learning control strategies were used in order to compare and obtain the best control law. Periodic forcing was able to significantly decrease the external separation point and the recirculation area, and to move up the internal separation point. Machine learning control was employed to search for new and more effective closed-loop control laws. Three MLC cases were studied: multifrequency open-loop, sensor-based and non-autonomous. Only twelve generations with 100 individuals were needed to converge towards a control law which outperforms periodic forcing. Non- 


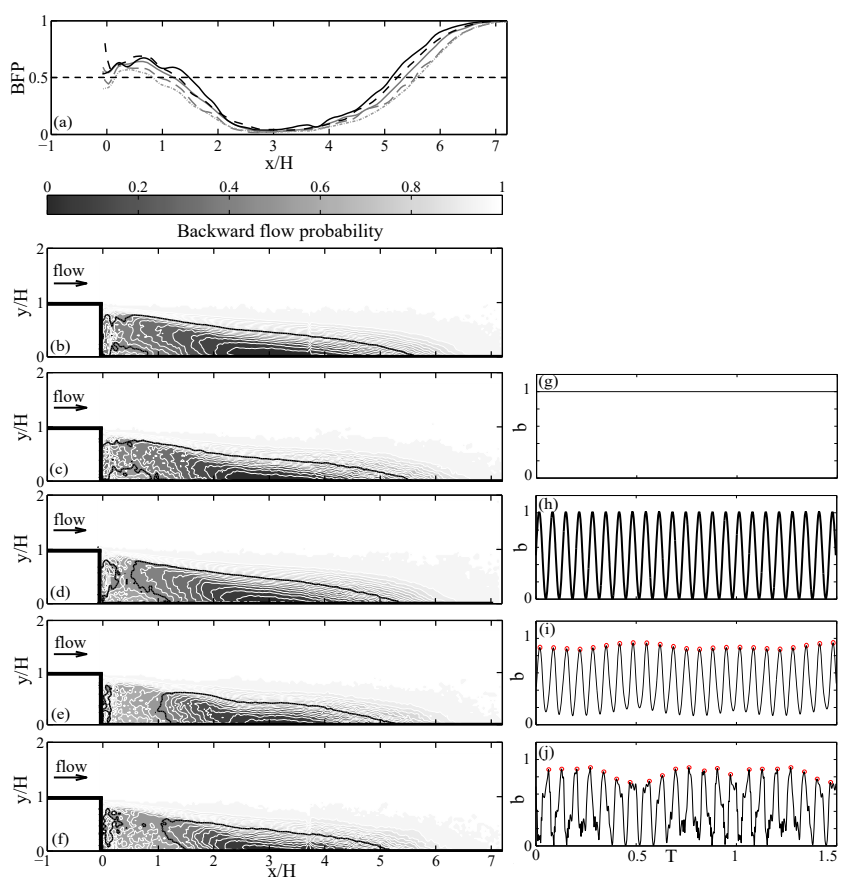

FIGURE 5. BACKWARD FLOW PROBABILITY. (a) BFP PROFILE EXTRACTED AT $y / \mathrm{H}=0.07$, FLOW PROBABILITY FIELD: (b) UNFORCED FLOW: DOT-DASHED GRAY LINE IN FIG.(a), (c) MLC SENSOR-BASED: DASHED GRAY LINE IN FIG.(a), (d) OPTIMAL PERIODIC FORCING: SOLID GRAY LINE IN FIG.(a), (e) MLC MULTI-FREQUENCY: DASHED BLACK LINE IN FIG.(a), (f) MLC NON-AUTONOMOUS: SOLID BLACK LINE IN FIG.(a), (g) CONTROL LAW SIGNAL MLC SENSOR-BASED, (h)CONTROL LAW SIGNAL OPTIMAL PERIODIC FORCING, (i) CONTROL LAW SIGNAL MLC MULTI-FREQUENCY,(j) CONTROL LAW SIGNAL MLC NON-AUTONOMOUS.

autonomous control showed to be the best control law strongly modifying all analyzed parameters. The resulting flows were investigated with real-time PIV. The study suggests that MLC can effectively explore and optimize new feedback actuation mechanisms and we anticipate MLC to be a game changer in turbulence control.

\section{ACKNOWLEDGMENT}

This work was carried out within the framework of the CNRS Research Federation on Ground Transports and Mobility, in articulation with the ELSAT2020 project supported by the European Community, the French Ministry of Higher Education and Research, the Hauts de France Regional Council. The authors gratefully acknowledge the support of these institutions.

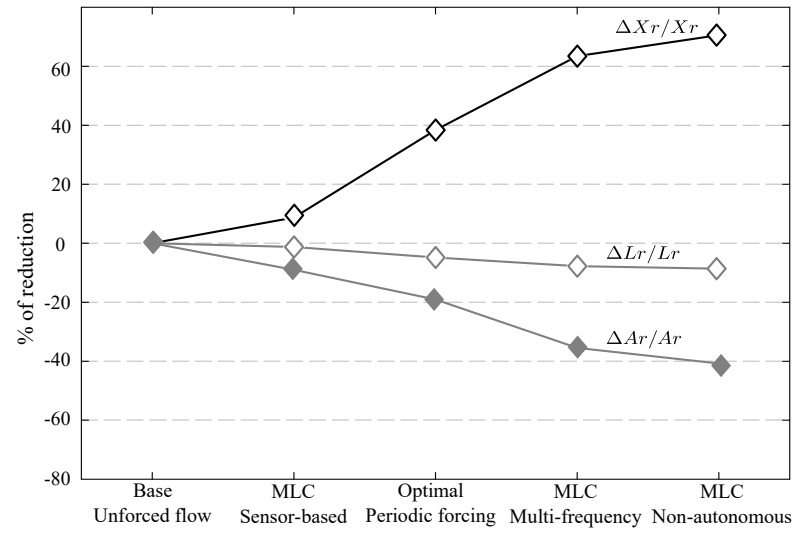

FIGURE 6. SUMMARY OF SEPARATION POINT AND REGION FOR ALL STUDIED CASES. $\diamond$ INTERNAL SEPARATION POINT $\mathrm{Xr}, \diamond$ EXTERNAL SEPARATION POINT Lr, $\diamond$ RECIRCULATION AREA Ar. VARIATIONS ARE IN \%

\section{REFERENCES}

[1] Chun, K.B., Sung, H.J. 1998 Visualization of a locallyforced separated flow over a backward-facing step. Experiments in Fluids, 25, 133-142.

[2] Hung, L., Parviz, M., Kim. J. 1998 Direct numerical simulation of turbulent flow over a backward-facing step J. of Fluid Mech., 330, 349-374.

[3] Beaudoin,J-F., Cadot, O., Aider,J-L. and Wesfreid,J.E. 2004 Three-dimensional stationary flow over a backwardsfacing step. European Journal of Mechanics, 38, 147-155.

[4] Aider,J-L., Danet,A., and Lesieur,M. 2007 Large-eddy simulation applied to study the influence of upstream conditions on the time-dependant and averaged characteristics of a backward-facing step flow. Journal of Turbulence, 8.

[5] Chun, K.B., Sung, H.J. 1996 Control of turbulent separated flow over a backward-facing step by local forcing. Experiments in Fluids, 21, 417-426.

[6] Yoshioka, S., Obi, S., Masuda, S. 2001 Turbulence statistics of periodically perturbed separated flow over a backward-facing step. International Journal of Heat and Fluid Flow, 22, 393-401.

[7] Yoshioka, S., Obi, S., Masuda, S. 2001 Organized vortex motion in periodically perturbed turbulent separated flow over a backward-facing step. International Journal of Heat and Fluid Flow, 22, 301-307.

[8] Dejoan A., Leschziner M.A. 2004 Large eddy simulation of periodically perturbed separated flow over a backwardfacing step International Journal of Heat and Fluid Flow, 25, 581-592.

[9] Bhattacharjee, S., Scheelke, R., Troutt, T.R. 1985 Modification of Vortex Interactions in a Reattaching Separated Flow 
Experiments in Fluids, 25, 133-142.

[10] Sipp, D., Marquet, O., Meliga, P., Barbagallo, A. 2010 Dynamics and control of global instabilities in open flows: a linearized approach. Appl. Mech. Rev, 63, 030801.

[11] Gautier, N., Aider J.-L., Duriez, T., Noack, B. R., Segond, M., Abel, M. 2015 Closed-loop separation control using machine learning J. Fluid Mech., 770, 442-457.

[12] Debien, A., von Krbek, K. A. F. F., Mazellier, N., Duriez, T., Cordier, L., Noack, B. R., Abel, M. W., Kourta, A. 2016 Closed-loop separation control over a sharp-edge ramp using genetic programming. Experiments in Fluids, 57 (40), $1-19$.

[13] Parezanovic, V., Cordier, L., Spohn, A., Duriez, T., Noack, B. R., Bonnet, J.-P., Segond, M., Abel, M., Brunton, S. L. 2016 Frequency selection by feedback control in a turbulent shear flow. Experiments in Fluids, 25, 133-142.

[14] De Brederode, V., Bradshaw, P. 1978 Influence of the side walls on the turbulent centerplane boundary-layer in a square duct. Trans. ASME I: J. Fluids Eng., 100, 91-96.

[15] Chovet, C., Lippert, M., Keirsbulck, L. and Foucaut, J.M. 2016 Dynamic characterization of piezoelectric microblowers for separation flow control. Experiments in Fluids, 25, 133-142.

[16] Duriez, T., Brunton, S. L. and Noack, B. R. 2016 Machine Learning Control - Taming Nonlinear Dynamics and Turbulence. Series Fluid Mechanics and Its Applications, vol. 116. Springer.

[17] Simpson, R.L. 1996 Aspects of turbulent boundary-layer separation. Prog.Aerospace Sci, 32, 457-521.

[18] Li, Z., Gao, N., Bai, H. 2015 Effect of the periodic perturbations on the wall pressure downstream of a backward facing step. International Conference on Fluid Mechanics, 25, 000-000.

[19] Nadge, P.M. and Govardhan, R.N. 2007 High Reynolds number flow over a backward-facing step: structure of the mean separation bubble. Exp. Fluids, 55:1657. 